\title{
Magnetic Reconnection and Transient Phenomena in Accretion Driven Systems
}

\section{P.J. Meintjes ${ }^{* \dagger}$}

Department of Physics, University of the Free State, Bloemfontein, 9300, South Africa

E-mail: MeintjPJ@ufs.ac.za

In this presentation I review some aspects of magnetohydrodynamics in astrophysical environments that can give rise to transient thermal and non-thermal emission. I focus specifically on the magnetic transport and reconnection in accretion driven systems, especially the role it may play in the production of transient multi-wavelength emission in these systems. The discussion related to magnetic reconnection starts with a brief historical overview, after which some of the latest results are discussed. It is shown that magnetic reconnection can indeed be a serious contender for the driving mechanism behind the transient burst-like emission in many accretion driven environments, from galactic to extra-galactic sources.

4th Annual Conference on High Energy Astrophysics in Southern Africa

25-27 August, 2016

Cape Town, South Africa

\footnotetext{
* Speaker.

${ }^{\dagger}$ The author expresses his appreciation towards SAAO for hosting this conference in 2016.
} 


\section{Introduction}

This discussion is based upon a historical overview ([1]) which just emphasizes some of the pioneering work that laid the foundation for our current understanding of magnetic reconnection as a mechanism driving particle heating and acceleration in astrophysical environments. It was suggested ([2,3]) that the reconnection of magnetic fields in X-points could be a source of particle heating and possibly acceleration. The interest of early researchers in X-points was probably due to the fact that a magnetic field inhibits particle acceleration unless there was a component of the electric field parallel to the magnetic field. Since it was thought that thermal electrons will short out these fields at large, it was thought that electric fields in localised regions such as X-points (magnetic null regions) could accelerate particles at least locally. It was pointed out ([4]) that Solar flares could be explained in terms of Ohmic heating in current sheets of a few metres in thickness. It has been pointed out ([5]) that a current sheet can occur when magnetic fields collapse near X-points. After this groundbreaking work, P.A. Sweet ([6, 7]) and E.N. Parker ([8]) developed simple steadystate MHD theories for the reconnection of magnetic fields and dissipation of magnetic energy in current sheets. It was proposed that when magnetized fluid zones of opposite polarity approach one another, a narrow "colliding layer" forms around the X-point and equipartition of magnetic and gas pressure in the reconnection zone demands that fluid gets pushed out of the "colliding layer" where the fields reconnect. It was in fact E.N. Parker that used the phrase "reconnection of magnetic fields" first where magnetic fields get "annihilated" ([9]). Parker applied the Sweet-Parker model, as it became known, to solar flares and found that the release in energy is about two orders of magnitude too slow to explain the flaring events - hence the Sweet-Parker model became known as the slow model. In calculating the reconnection rate, both Sweet and Parker assumed a plasma resistivity quantified by Spitzer (e.g [10]), which may not be the case in flaring coronal plasmas. However, an alternative model was developed that could produce faster reconnection rates and energy release ([11]), even if Spitzer's plasma conductivity is assumed. This model, the so-called Petschek model, became known as the faster model for reconnection. However, it should be stressed that even the Petschek model, albeit significantly faster than the Sweet-Parker process, also fails to explain the rapid energy release in solar flares. One of the very important developments that occurred more or less contemporaneous was the discovery of the tearing mode instability ([12]), which is a vital ingredient in the physics of magnetic reconnection. A unified model for reconnection was developed by ([13]) in which both these models, namely the Sweet-Parker and Petschek models are special cases (e.g. [1] for a detailed discussion). In this introduction, just a very brief historical account has been presented to celebrate some of the pioneers that established the fundamentals of magnetic reconnection, although there have been many more who contributed to our understanding of this phenomenon (see e.g. [1, 14, 15] for in-depth discussions).

The paper will be structured as follows: In the following section, a basic discussion of magnetic field transport in astrophysical plasmas will be presented. The basic properties of magnetic reconnection described in both the Sweet-Parker and Petschek models will be reviewed and it will be illustrated why they both failed to explain the time scale of energy release in astrophysical environments. In the section following that, it will be shown that MHD turbulence can indeed speed-up the reconnection process, with acceleration of particles resulting in power-law spectra. This is followed by a discussion of the possible relation between magnetic reconnection and fast transient 
phenomena in astrophysical environments, after which concluding remarks are presented.

\section{Magnetic Transport and Reconnection: The Sweet-Parker and Petschek models}

Magnetic reconnection involves the diffusion (e.g. [1, 15]) of magnetic fields of opposite polarity, partly "frozen" into a plasma until they meet, creating a zone of zero magnetic field or "null zone" when the fields annihilate. The main driver of magnetic transport and subsequent reconnection is the magnetic diffusivity, which is determined by the conductivity of the fluid. The magnetic induction law, applied to a fluid, is

$$
\frac{\partial \mathbf{B}}{\partial t}=\nabla \times(\mathbf{v} \times \mathbf{B})+\eta \nabla^{2} \mathbf{B}
$$

where $\eta=c^{2} / 4 \pi \sigma$ represents the resistive diffusivity, with $\sigma$ representing the plasma electrical conductivity. The second term, as expected, determines the rate at which magnetic fields can diffuse through a conducting fluid, or plasma. It can be shown that the magnetic transport can be quantified by the so-called magnetic Reynolds number, i.e.

$$
R_{\mathrm{m}}=\frac{L V}{\eta}
$$

or equivalently, the so-called Lundquist number, which is just the ratio of the resistive diffusivity timescale to the timescale an Alfvén wave will cross the plasma (e.g. [16]), i.e.

$$
\begin{aligned}
S & =\frac{L V_{\mathrm{A}}}{\eta} \\
& =\frac{\tau_{\mathrm{res}}}{\tau_{\mathrm{A}}}
\end{aligned}
$$

which can be written as ([16])

$$
S=2 \times 10^{-2} L B T_{\mathrm{e}}^{3 / 2} n_{\mathrm{e}}^{-1 / 2}\left(\frac{\lambda}{10}\right)^{-1},
$$

where $L, B, T_{\mathrm{e}}, n_{\mathrm{e}}$ and $\lambda$ represent the length scale, magnetic field, electron temperature, density and Coulomb logarithm of the plasma respectively. For various astrophysical plasmas, the Lundquist number is anything between $10^{9}-10^{15}$ (e.g. $[1,16,17]$ ), which implies that the resistive diffusion timescale of magnetic fields through a highly conducting plasma is extremely slow. This will influence the reconnection timescale and subsequent energy release timescale, for both the SweetParker and Petschek mechanisms. For the Sweet-Parker process, the dimensionless reconnection rate scales as $\left(v_{\text {rec }} / v_{\mathrm{A}}\right) \propto\left(1 / S^{1 / 2}\right)$, whereas for the Petschek family of solutions, the reconnection rate scales as $\left(v_{\text {rec }} / v_{\mathrm{A}}\right) \propto(1 / \ln S)$, which shows that the Petschek process is faster even for large Lundquist number plasmas (see e.g. [16]). The widening of the outlet funnels in X-point reconnection, described by the Petschek model, prevents the formation of shock waves in the outflow that will slow down further reconnection. However the X-point geometry implies local anomalous resistivity in the plasma, which is rather difficult to reconcile with astrophysical plasmas in general.

It has been shown that current sheets can be prone to the development of the tearing mode instability ([12]), which is a resistive instability breaking up the current sheet into magnetic islands. 


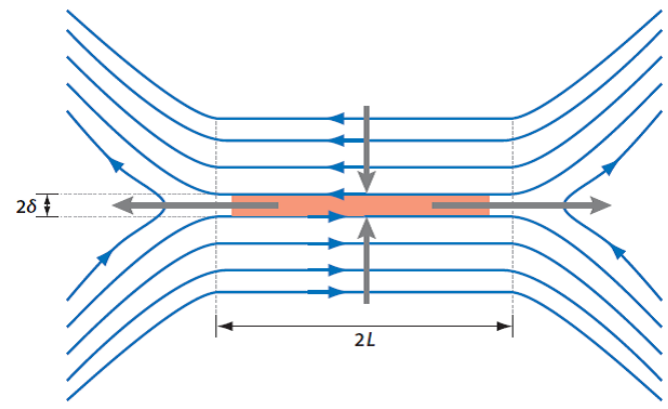

Figure 1: Reconnection in the Sweet-Parker (slow) Figure 2: Reconnection in the Petschek (fast) model. Adopted from [16].

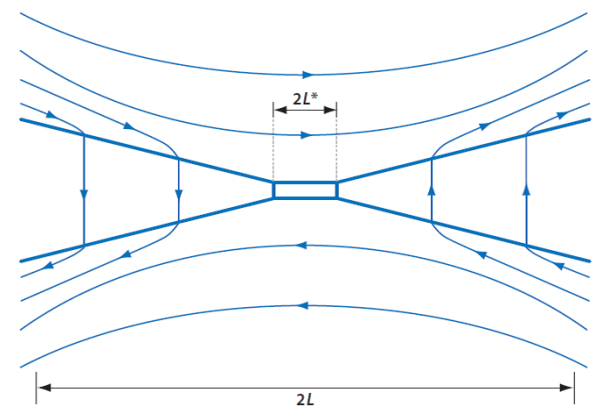
model. Adopted from [16].

This is confirmed in particle-in-cell (PIC) simulations of magnetic reconnection, which provides yet another independent tool to investigate this phenomenon numerically ([20, 21]).

The driving mechanism for the development of the tearing mode instability is the $\mathbf{j} \times \mathbf{B}$ force acting in different directions above and below the neutral sheet (e.g. [17]), where $\mathbf{j}$ represents the electric current in the current sheet driven by the electric field $\mathbf{E} \propto \mathbf{v}_{\text {rec }} \times \mathbf{B}$ above and below the neutral sheet. From an ideal MHD perspective pressure equilibrium will be established between the merging magnetic fields and plasma, but anomalous resistivity may result in slippage of the magnetic field through the plasma resulting in reconnection and the tearing mode instability. Resistivity is the highest in the null points where the magnetic fields reconnect. An additional consequence of the development of the tearing mode instability, is the subsequent formation of the coalescence instability (e.g. $[18,19])$ which merges separate magnetic islands into one. The coalescence instability completes the collapse initiated by the tearing mode instability with subsequent release of the bulk of the energy in the current sheet.

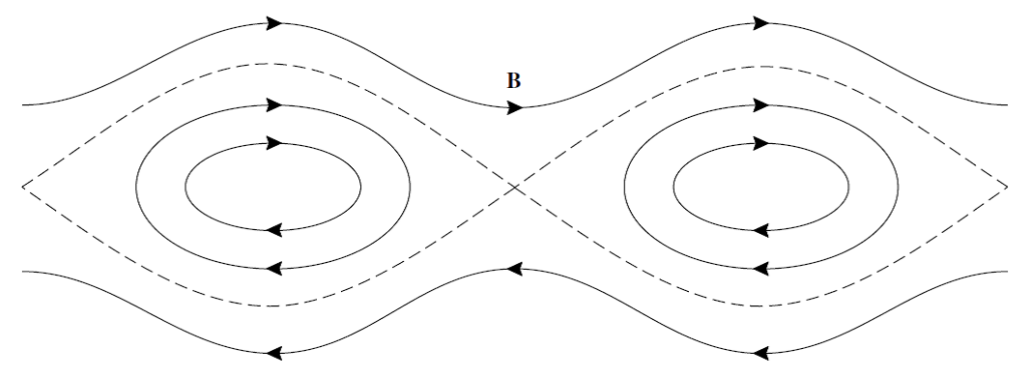

Figure 3: The tearing model instability. Adopted from [17].

Current sheets with anomalous resistivity provide the potential for particle acceleration in reconnection events. In a detailed MHD study of particle acceleration in magnetic reconnection, it has been shown ([22]) that the electric field in the current sheet (perpendicular to the page in the geometries depicted in Fig 1-3) will have the following magnitude

$$
\mathbf{E}=-\frac{c}{4 \pi \sigma} \frac{d B}{d x}+\frac{v_{\mathrm{rec}} B}{c} .
$$


For regions far from the X-point, where $\frac{d B}{d x} \rightarrow 0$, the electric field is dominated by $\mathbf{E} \sim v_{\operatorname{rec}} B / c$, while near the null X-point $B \rightarrow 0$, with the dominant component now being $\mathbf{E} \sim-\eta / c \frac{d B}{d x}$, with $\eta=c^{2} / 4 \pi \sigma$. Anomalous resistivity in current sheets is determined by the condition (see e.g. [22])

$$
\frac{B \sigma}{n_{\mathrm{e}}}>10^{2}
$$

where $B, \sigma$ and $n_{\mathrm{e}}$ represent the magnetic field, the conductivity and electron density respectively. It is obvious from the condition above that anomalous resistivity will play a significant role in current sheets developing in highly magnetic and low density plasmas. For a current sheet satisfying this condition, strong electric fields will be induced that has the potential to accelerate particles to high energies. It has been shown that for induced electric fields exceeding the so-called Dreicer field ([23, 24]), i.e. $E_{\mathrm{D}} \sim 10^{-11} \ln \Lambda n_{\mathrm{e}} T_{\mathrm{e}}^{-1}$ statvolt $\mathrm{cm}^{-1}$ (e.g. [19]), with $\ln \Lambda \sim 10-20$ being the Coulomb logarithm and $n_{\mathrm{e}}$ and $T_{\mathrm{e}}$ representing the electron density and temperature respectively, a run-away particle acceleration process will occur in the current sheet. The momentum gained by these accelerated particles will be at the cost of their transverse momentum, resulting in highly focussed ([25]) relativistic particle beams (see Fig.4), which may have implications for rapid variability in several classes of astrophysical objects.

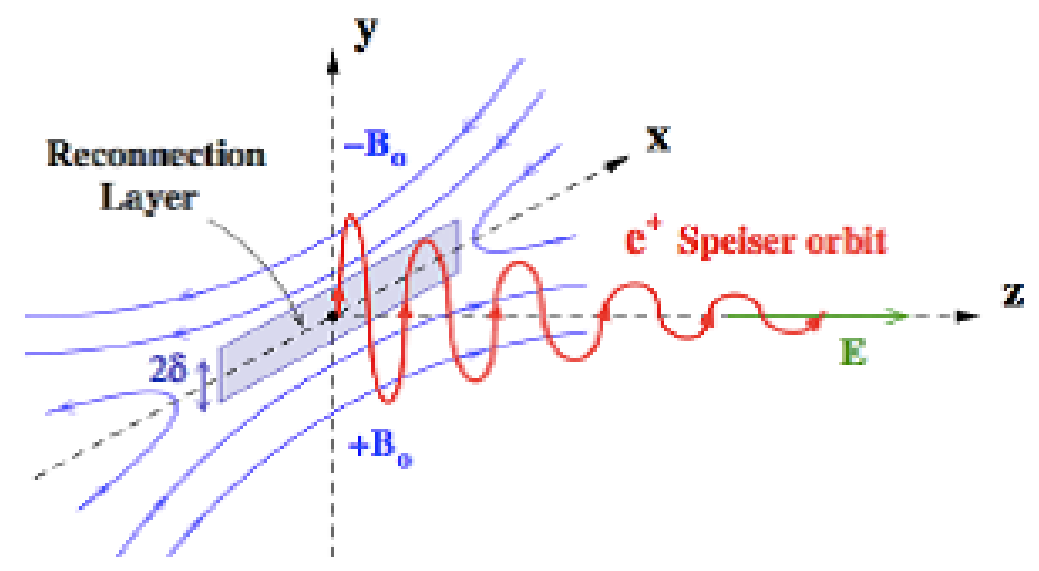

Figure 4: Reconnection producing highly focussed particle beams. Adopted from [25].

A consequence of reconnection in low density plasmas is the fact that the reconnection rate can dramatically increase since the reconnection speed $v_{\text {rec }} \rightarrow v_{\mathrm{A}}$ (Alfvén velocity), with $v_{\mathrm{A}}=$ $B / \sqrt{4 \pi \rho} \rightarrow c$ for highly magnetic low density plasmas, i.e. $\beta=\left(8 \pi n_{\mathrm{e}} k T / B^{2}\right)<<1$ (low-beta plasmas). The fact that $v_{\text {rec }} \rightarrow c$ has very important conseqences for particle acceleration to very high energies in $\beta<<1$ astrophysical environments.

\section{Collisionless Turbulent Reconnection}

More recent developments in the theory of magnetic reconnection (e.g. [26]) investigated the influence of magnetic fluctuations (or turbulence) on the reconnection rate. These authors showed that a lower limit on the reconnection speed scales as $v_{\text {rec }}=v_{\mathrm{A}} S^{-3 / 16} M^{3 / 4}$, with an upper limit 
on the reconnection speed scaling as $v_{\text {rec }}=v_{\mathrm{A}} M^{2}$, where $v_{\mathrm{A}}$ is the Alfvén speed, $S$ the Lundquist number and $M$ the Mach number of the turbulence field. They (i.e. [26]) showed that the fraction of the magnetic energy that is channeled into electron heating scales as $\propto S^{-2 / 5} M^{8 / 5}$ whereas the thickness of the current sheet scales as $\propto S^{-3 / 5} M^{-2 / 5}$, while a significant fraction of the magnetic energy goes into the generation of high frequency Alfvén waves that may provide a mechanism for particle acceleration. These authors showed that the turbulence field will have the effect that reconnection can occur simultaneously over a longer length span in the current sheet, which essentially speeds-up signficantly the overall reconnection rate (e.g. see Figure 5.).
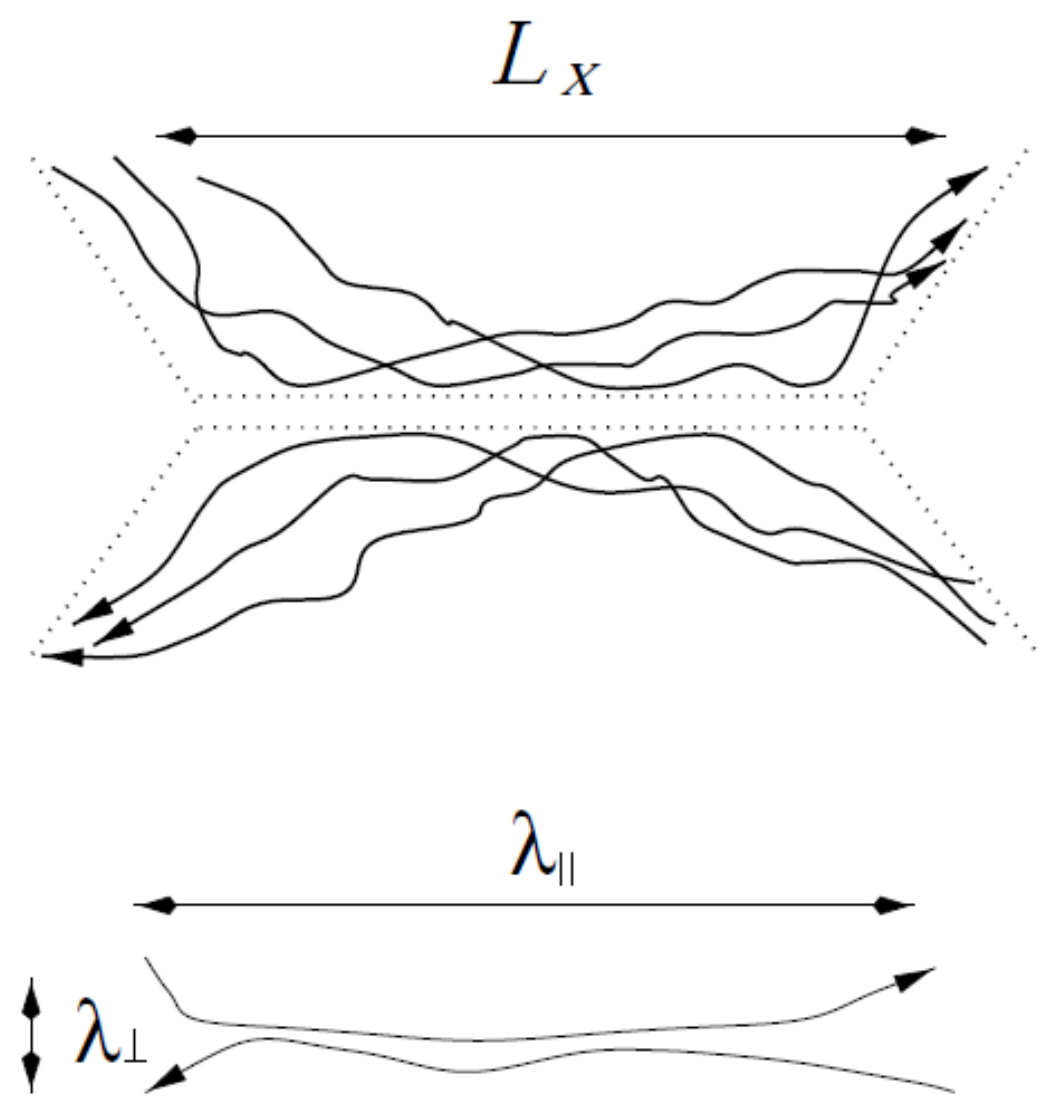

Figure 5: Reconnection in a turbulent magnetic field, leading to faster overall reconnection. The reconnection occurs simultaneously in several localized dissipation zones of dimension $\lambda_{\|}<<L_{X}$. Adopted from [26].

In the presence of a stochastic turbulent magnetic field the localized magnetic dissipation occurs over length scales $\lambda_{\|}<<L_{X}$ where the field direction deviates from its original direction over a width increment $\lambda_{\perp}=\eta / v_{\text {rec,local }}$. However, this can occur simultaneously in several locations spread over the entire reconnection zone with length $L_{\mathrm{X}}$. The additional bonus is that reconnection is determined by the plasma turbulence and independent, or very weakly dependent, on the plasma resistivity. Turbulence can be inferred in all astrophysical environments, over all length scales (e.g. Figure 6) (see e.g.[27, 28]). 


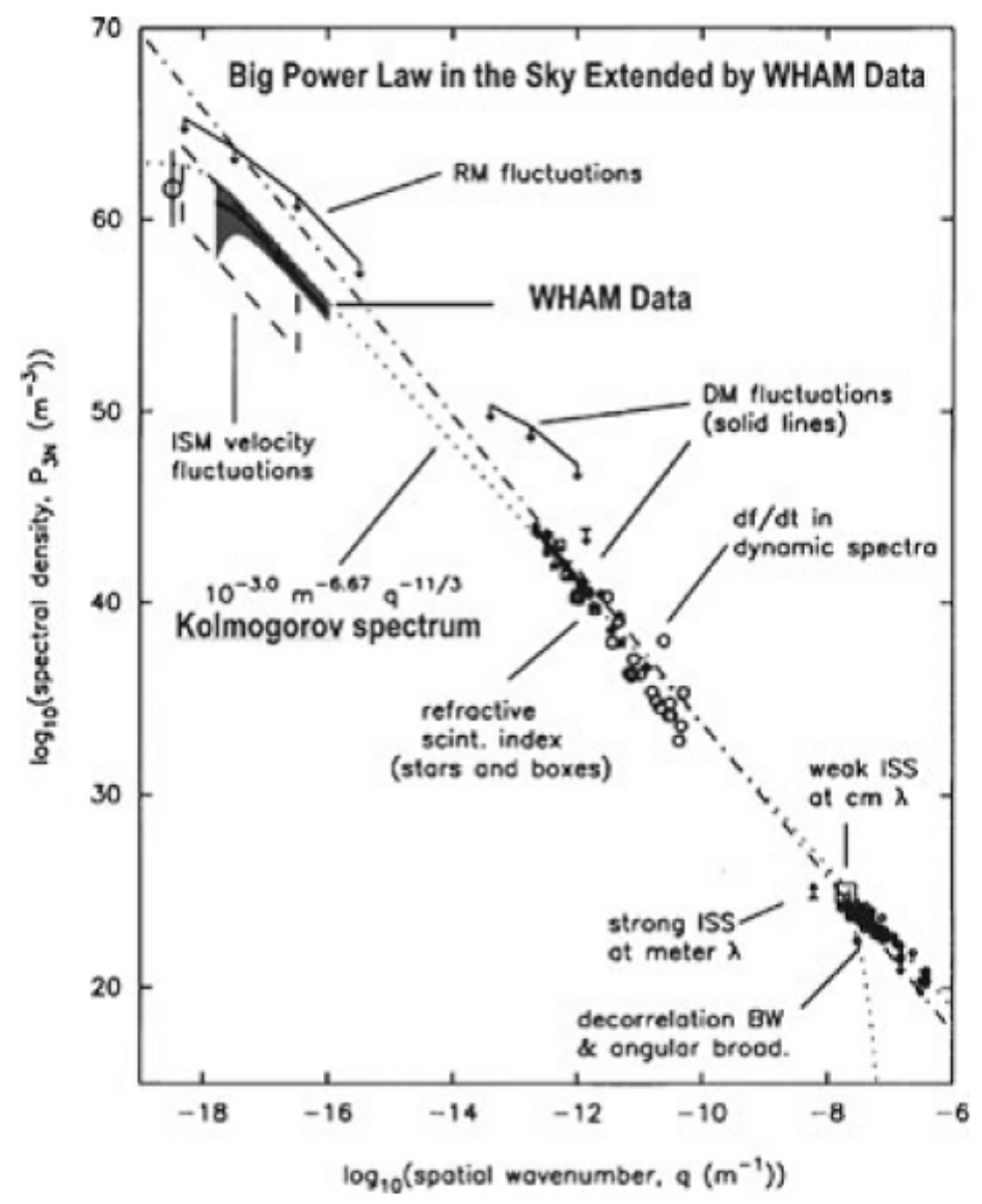

Figure 6: Turbulence is an inherent phenomenon in all astrophysical environments over all length scales, resulting in "The Big Power-Law in the Sky". The slope corresponds to a Kolmogorov spectrum. Adopted and adapted from $[27,28]$ respectively.

\section{Particle Acceleration in Turbulent Collisionless Reconnection}

It has been pointed out (e.g. $[29,30])$ that particle acceleration in turbulent approaching magnetic fields is essentially a first-order Fermi process, since particles like electrons attached to the field lines essentially see the approaching turbulent fields as magnetic scattering centers. In this first-order Fermi process the energy gain per scattering is essentially

$$
\left\langle\frac{\Delta \varepsilon}{\varepsilon}\right\rangle=\frac{4}{3} \frac{v_{\mathrm{rec}}}{c}
$$

which results in a total energy gain after a round trip between scattering centers

$$
\frac{\varepsilon}{\varepsilon_{\circ}}=1+\frac{8}{3} \frac{v_{\mathrm{rec}}}{c} .
$$

This first-order Fermi process has been verified in subsequent 2D and 3D numerical simulations (e.g. [31, 32]). It can be shown analytically that the particle spectrum from a first-order 
process will be a power-law spectrum (e.g. [33]), which is confirmed by numerical simulations concerning particle acceleration in reconnection events ([25]). The argument is based on the energy gained by each particle in a scattering being $\varepsilon=\beta \varepsilon_{\circ}$, where $\beta$ is a fraction of the initial energy $\varepsilon_{\circ}$. After $\mathrm{m}$ - scatterings we have $\varepsilon=\beta^{m} \varepsilon_{\circ}$, where the total number of accelerated particles which gained energy are $N=P^{m} N_{\circ}$, where $P^{m}$ represents the probability that the particles stayed in the accelerator zone for the full quota of $\mathrm{m}$-scatters. Therefore, it can be shown that

$$
\begin{aligned}
\frac{N}{N_{\circ}} & =P^{\frac{\ln \left(\varepsilon / \varepsilon_{\circ}\right)}{\ln \beta}} \\
& =\left(\frac{\varepsilon}{\varepsilon_{\circ}}\right)^{\frac{\ln P}{\ln \beta}}
\end{aligned}
$$

which can readily be converted to a spectrum

$$
N(\varepsilon) d \varepsilon=\text { const } \varepsilon^{(-1+(\ln P / \ln \beta))} d \varepsilon .
$$

It has been shown (e.g. [29]) that the probability $P=\left(1-4\left(v_{\text {rec }} / c\right)\right)$, with $\beta=\left(\varepsilon / \varepsilon_{\circ}\right)=(1+$ $\left.8 / 3\left(v_{\text {rec }} / c\right)\right)$, which in the limit $\left(v_{\text {rec }} / c\right)<1$ results in $N(\varepsilon) d \varepsilon \propto \varepsilon^{-5 / 2} d \varepsilon$ ([29]). However, for first-order Fermi acceleration occuring in strong shocked plasmas it can be shown that the powerlaw index for Fermi acceleration can scale as $N(\varepsilon) d \varepsilon \propto \varepsilon^{-s} d \varepsilon$, with $s=(r+2) /(r-1)$ and $r$ representing the compression ratio across the shock. For strong shocks $r \rightarrow 4$. This results in a typical spectrum $N(\varepsilon) d \varepsilon \propto \varepsilon^{-2} d \varepsilon$ (e.g. [33]). This means that the first-order Fermi acceleration spectrum associated with reconnection in turbulent plasmas, namely $N(\varepsilon) \propto \varepsilon^{-(5 / 2)}$, may be distinguishable from the typical acceleration spectrum produced by a first-order Fermi process in strong shocks, which is $N(\varepsilon) \propto \varepsilon^{-(2)}$ ([29]).

\section{Reconnection in Accretion Driven Astrophysical Environments}

It has been shown that the flaring activity of micro quasars (GRS1915+105) and general Kerr black holes in e.g. active galactic nuclei (AGN), can be reconciled with magnetic reconnection at the base of the jet as well the generation of plasmoids in the jet (e.g.[29, 30, 34]). These authors showed that reconnection can indeed account for the non-thermal radio synchrotron spectra observed in these sources during flares. It has been shown (e.g. [26]) that magnetohydrodynamic (MHD) turbulence provides the theoretical framework to investigate magnetic reconnection in magnetized plasmas. It is believed that MHD turbulence (e.g. [35, 36, 37]) plays a fundamental role in the accretion disc viscosity that drives, for example, dwarf nova eruptions and other transient phenomena associated with the magnetorotational instability (MRI) (e.g. [38, 39, 40]).

\subsection{Reconnection and the Dwarf Nova Phenomenon: Magnetic Advection}

The recent detection of non-thermal radio synchrotron emission during dwarf nova eruptions (e.g. [41]) may provide an interesting case to investigate the role that magnetic reconnection can play in these events. It has been pointed out (e.g. $[42,43,44])$ that the accretion discs of slow rotating compact objects in disc accreting systems may be unstable to the build-up of MHD turbulence outside the corotation radius. The differential rotation between the accreting compact object and the disc will result in the build-up of toroidal fields in the disc (see Figure 7) which will be unstable 
to the tearing mode instability, as well as the Parker instability ([45]), resulting in strong toroidal and vertical magnetic fields that can drive the magnetorotational instability (MRI) and reconnection. The reconnection can release magnetic energy in the form of disc heating and possibly also particle acceleraton. It has been shown $([42,43,44])$ that the build-up of magnetic energy in the disc may result in the Hartmann number $(M)$, i.e. the ratio of magnetic stresses to fluid viscosity, being $M \geq 1$, which will have a devastating effect on the azimuthal disc flow dynamics. These autors showed that for typical parameters for the disc magnetic field $(B)$, disk height $(H)$ and turbulent viscosity $\left(\nu_{\mathrm{T}}\right)$ and turbulent disc conductivity $\left(\sigma_{\mathrm{T}}\right)$, the Hartmann number for an unstable disc is

$$
M>1\left(\frac{B_{\text {disc }}}{100 \mathrm{G}}\right)\left(\frac{L}{H_{\text {disc }}}\right)\left(\frac{v_{\mathrm{T}}}{10^{13} \mathrm{~cm}^{2} \mathrm{~s}^{-1}}\right)^{-1 / 2}\left(\frac{\sigma_{\mathrm{T}}}{10^{7} \mathrm{~s}^{-1}}\right)^{1 / 2} .
$$

This implies a global break-down of the azimuthal flow profile of the disc outside the corotation radius, which will advect magnetic fields inwards during the dwarf nova outburst. This will also have consequences for the magnetic field of the compact object still frozen into the disc, which will be advected inward with the flow, providing the ideal conditions for magnetic reconnection and particle acceleration (see e.g. Figure 8).

(a) Coroniti model

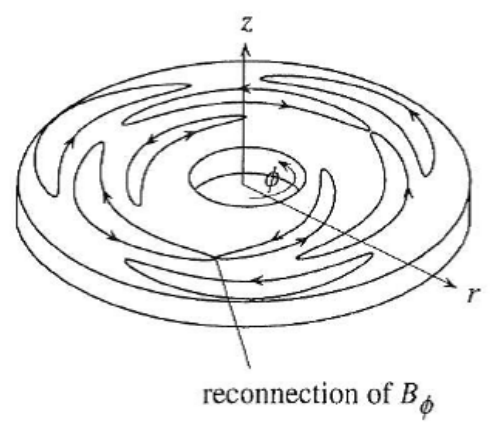

(b) Tout \& Pringle model

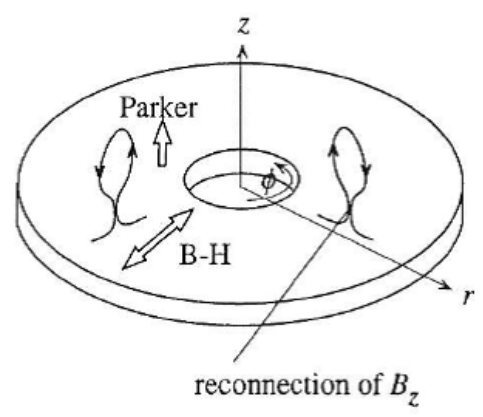

Figure 7: Turbulent magnetic field generation in an accretion disc. Strong toroidal fields will form (a) which will be subjected to the tearing mode and Parker instabilities, resulting in vertical magnetic fields (b) that drive the MRI instability as well as reconnection. This will drive disc heating and possible particle acceleration. Adopted from [1].

\subsection{Dwarf Nova Outbursts and Non-Thermal Synchrotron Flares}

In these events, the reconnection will produce magnetic bubbles ([46]) being centrifugally ejected from the binary system, expanding as they radiate through synchrotron radiation, i.e the so-called van der Laan process (e.g. [47]). It has been shown (e.g. [48]) that the continuous flaring activity of the novalike variable AE Aquarii could be described as a superposition of expanding synchrotron radiating bubbles. It can be shown that the peak flux from an expanding synchrotron radiating bubble, as well as the frequency where the peak emission occurs, can be expressed as

$$
\begin{aligned}
S(v, \rho)= & S_{\mathrm{mo}}\left(\frac{v}{v_{\mathrm{m}}}\right)^{5 / 2} \rho^{3} \\
& \times \frac{\left\{1-\exp \left[-\tau_{\mathrm{m}}\left(v / v_{\mathrm{mo}}\right)^{-(\delta+4) / 2} \rho^{-(2 \delta+3)}\right]\right\}}{1-\exp \left(-\tau_{\mathrm{m}}\right)},
\end{aligned}
$$



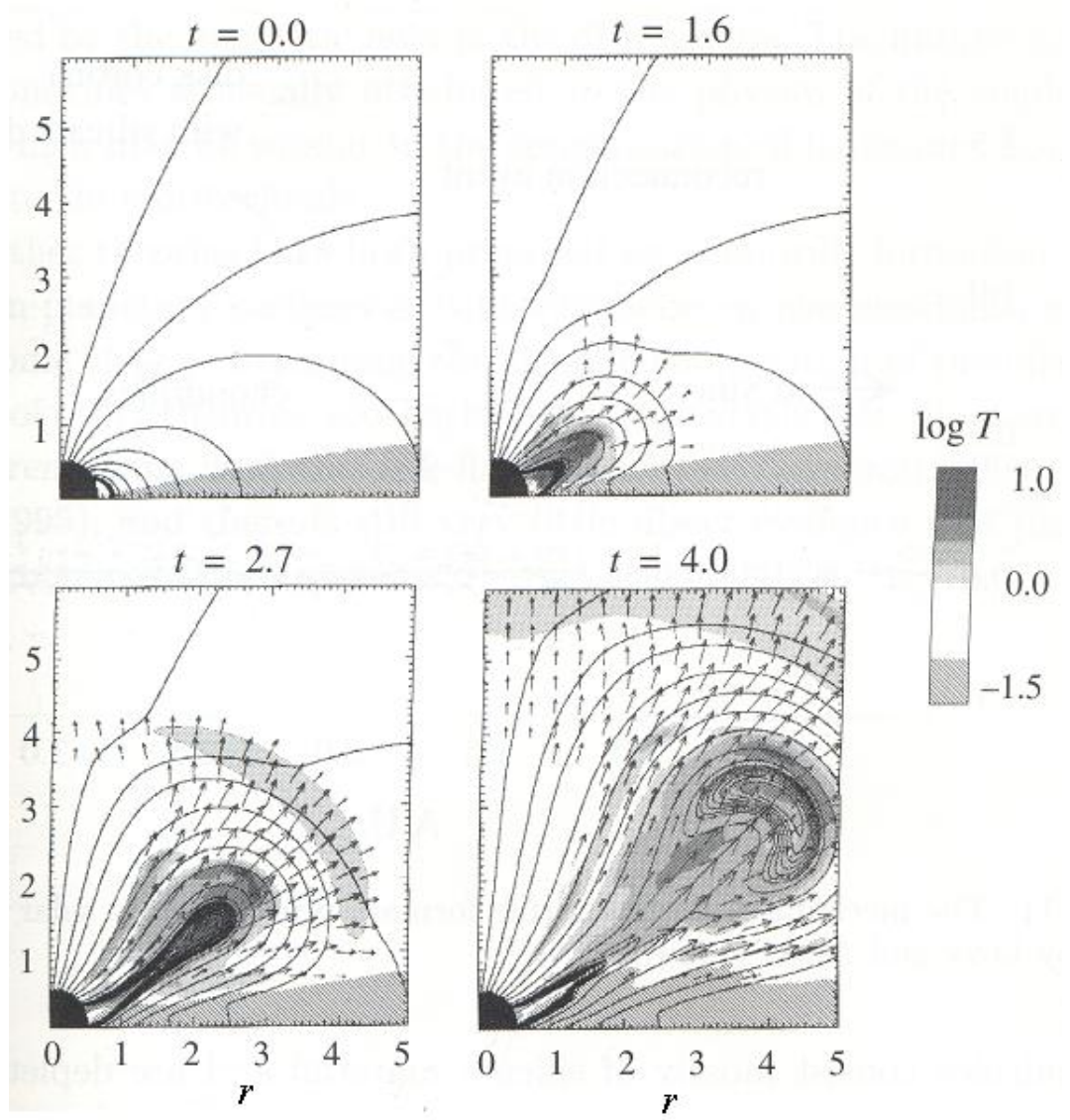

Figure 8: Magnetic advection during dwarf nova eruptions, leading to the formation of magnetic bubbles and subsequent reconnection. Adopted from [46, 1].

where $S_{\mathrm{m}}$ is the maximum flux density at the initial time $t_{\circ}, v_{\mathrm{m}}$ is the frequency at this maximum and $\tau_{\mathrm{m}}$ is the optical depth at this frequency, $\rho=\left(r / r_{\circ}\right)$ is a dimensionless parameter expressing the blob radius in relation to its initial radius and where $\delta$ is the electron spectral index. The frequency $v_{\mathrm{m}}$ depends on radius as

$$
v(\rho)=v_{\mathrm{m} \circ} \rho^{-(4 \delta+6) /(\delta+4)} .
$$

Therefore the non thermal flux, i.e. $S(v, \rho)$, asymptotically depends on $v$ and $\rho$ like

$$
S(v, \rho)=\left\{\begin{array}{l}
S(v) \rho^{3}, v \ll v_{\mathrm{m}}, \\
S(v) \rho^{-2 \delta}, v \gg v_{\mathrm{m}}
\end{array}\right.
$$

For single flare events we have

$$
\begin{aligned}
S_{\mathrm{m}, \circ}= & A_{1}\left[A_{2} \frac{\delta-1}{\delta+2}\left(\frac{E_{\mathrm{c}}}{1 \mathrm{MeV} V}\right)^{\delta-1} N_{\circ}\right]^{3 /(2 \delta+3)} \\
& \times\left[r_{\circ}^{(4 \delta+9) / 3} B_{\circ}^{(\delta+3) / 6}\right]^{3 /(2 \delta+3)} v_{\mathrm{m}}{ }^{(7 \delta+3) /(4 \delta+6)}
\end{aligned}
$$

with $A_{1}=3.3 \times 10^{6} \times 2^{-\delta / 2} \pi k / c^{2} D^{2}, A_{2}=\left(2.8 \times 10^{7}\right)^{(\delta+4) / 2} / 10^{11}$ and

$$
N_{\circ}=A_{3}\left[\frac{\delta+2}{\delta-1}\left(\frac{E_{\mathrm{c}}}{1 \mathrm{MeV}}\right)^{-(\delta-1)}\left(\frac{B_{\circ}^{2 \delta+9}}{r_{\circ}}\right)\right]^{1 /(\delta+5)},
$$


where $N_{\circ}$ represents the electron number density assuming equipartition between the magnetic and relativistic electron energy densities. In this equation the constant $A_{3}=\left[3.2 \times 10^{4} \times 2^{\delta / 2}\left(10^{11}\right)^{1 /(\delta+4)}\right]^{(\delta+4) /(\delta+5)}$. In Figure 9 the time evolution of the synchrotron flux from a single expanding cloud is illustrated.
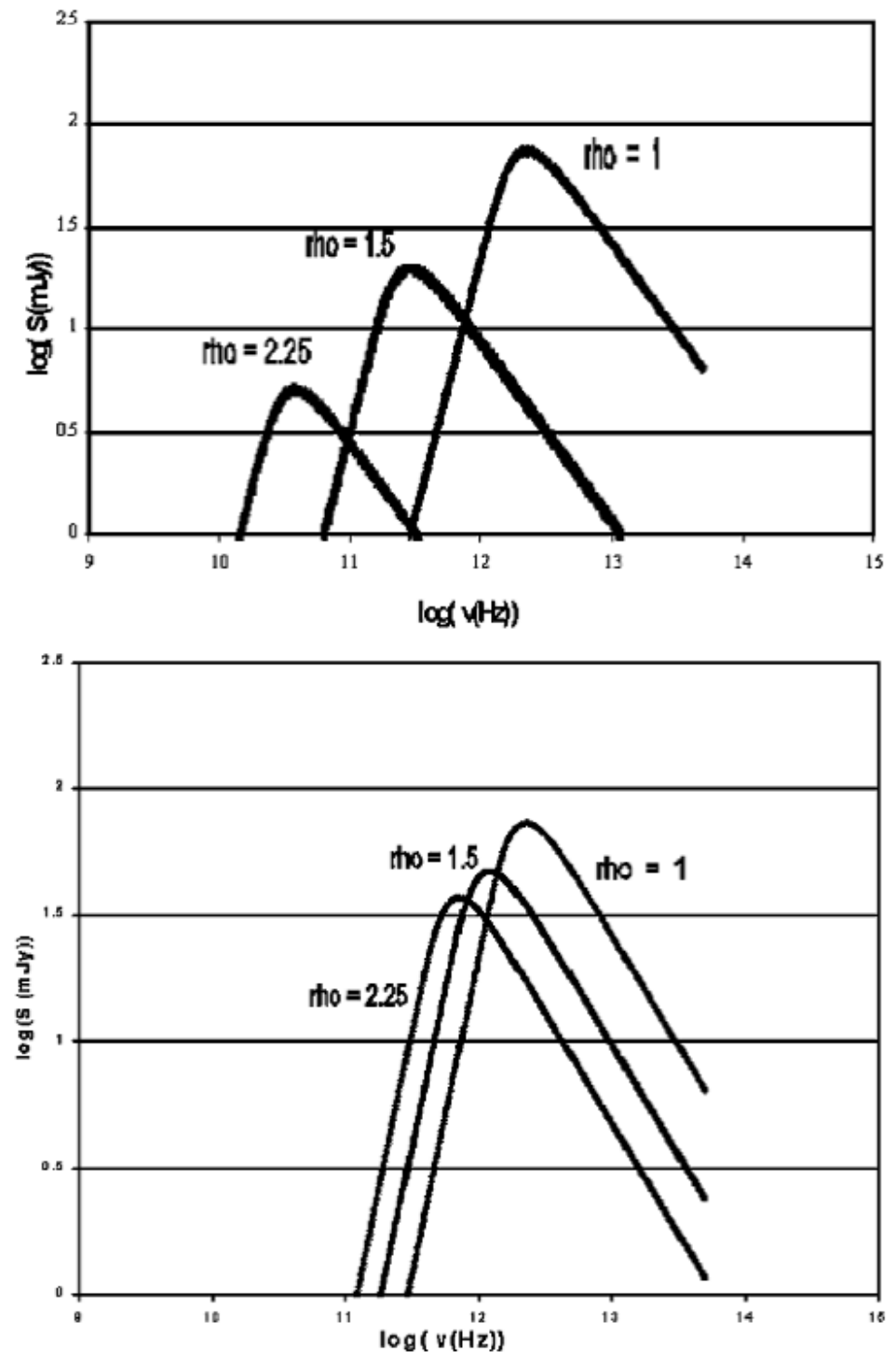

Figure 9: Synchrotron flux of a single synchrotron emitting cloud evolving with time - note how the peak flux evolves to lower frequencies as the cloud expands and cools. The top panel represents bubbles expanding adiabatically, while re-acceleration occurs in the bottom panel. Adopted from [49].

It can be shown that the initial flux of an expanding synchrotron bubble associated with a source at a distance of approximately $100 \mathrm{pc}$, is approximately (adopting $\delta=2.75$ ) 


$$
\begin{aligned}
S_{\mathrm{m}, \circ} \approx & 60\left[\left(\frac{\varepsilon_{\mathrm{e}}}{1 \mathrm{MeV}}\right)^{1.75}\left(\frac{r_{\circ}}{10^{9} \mathrm{~cm}}\right)^{6.67}\left(\frac{N_{\circ}}{10^{9} \mathrm{~cm}}\right)\left(\frac{B_{\circ}}{100 \mathrm{Gauss}}\right)^{0.958}\right]^{0.3529} \times \\
& \left(\frac{v_{\mathrm{m} \circ}}{60 \mathrm{GHz}}\right)^{1.3088} \mu \mathrm{Jy}
\end{aligned}
$$

with the frequency where the initial flux peaks being

$$
\nu_{\circ} \approx 60\left(\frac{\varepsilon_{\mathrm{e}}}{\mathrm{MeV}}\right)^{0.5185}\left(\frac{N_{\circ}}{10^{9} \mathrm{~cm}^{-3}}\right)^{0.2963}\left(\frac{r_{\circ}}{10^{9} \mathrm{~cm}}\right)^{0.2963}\left(\frac{B_{\circ}}{100 \mathrm{G}}\right)^{0.703} \mathrm{GHz}
$$

At frequencies below $v_{\circ}$ the flux spectrum will typically follow a self-absorbed $S_{v} \propto v^{\alpha}$ spectrum (e.g. [48, 49]).

Recent studies ([41]) of radio emission of several novalike variables, utilizing the Very Large Array (VLA), showed some dwarf novae during outburst being strong radio emitters. The flux levels range beween 30-200 $\mu \mathrm{Jy}$. It has been shown that for some sources, namely TT Ari and V603 Aql, a self-absorbed spectrum $S_{v} \propto v^{\alpha}$ has been observed, with the turnover frequency for V603 Aql of the order of $\sim 10 \mathrm{GHz}$. These detections provide new opportunities for the study of non-thermal emission during flares with the meerKAT telescope currently being developed in Southern Africa.

\section{Conclusion}

In this study a very brief summary of magnetic reconnection has been presented. It was shown that magnetic reconnection can provide a very attractive theoretical framework to evaluate transient phenomena in astrophysical environments. It has been shown that magnetic reconnection can be a very efficient process to convert magnetic energy into thermal energy and particle acceleration in turbulent plasmas. The association of dwarf nova eruptions with MHD turbulence provides an opportunity to investigate whether turbulent reconnection during dwarf nova eruptions can be responsible for expanding synchrotron emitting bubbles, which radiate as they expand. It was shown that this process may indeed be associated with the non-thermal radio emission detected recently of several dwarf novae during outbursts.

\section{References}

[1] E. Priest \& T. Forbes, Magnetic Reconnection: MHD theory and application, Cambridge Univ. Press, Cambridge (2000)

[2] R.G. Giovanelli, A Theory of chromospheric flares, Nature 15881 (1946)

[3] F. Hoyle, Some recent researches in Solar Physics (Cambridge Univ. Press, Cambridge) (1949)

[4] T.G. Cowling, Solar Electrodynamics, The Sun, ed. G. P. Kuiper (University of Chicago Press, Chicago) pp. 532-591 (1953) 
[5] J.W. Dungey, Conditions for the occurrence of electrical discharges in astrophysical systems, Phil. Mag. 44 725-738 (1953)

[6] P.A. Sweet, The neutral point theory of solar flares, Electromagnetic phenomena in cosmic plasmas, IAU Symposium 6 (ed. B. Lehnert) (Cambridge Univ. Press, London) pp. 123-34 (1958a)

[7] P.A. Sweet, The production of high energy particles in solar flares, Nuovo Cimento Suppl. 8, Ser. X, 188-196 (1958b)

[8] E.N. Parker, Sweet's mechanism for merging magnetic fields in conducting fluids, J. Geophys. Research 62 509-520 (1957)

[9] E.N. Parker, The solar flare phenomenon and theory of reconnection and annihilation of magnetic fields, Astrophys. J. Suppl. 8 177-211 (1963)

[10] L. Spitzer, Physics of fully ionized gases (Interscience, New York) (1962)

[11] H.E. Petschek, Magnetic field annihilation, in Physics of Solar Flares ed. W.N. Hess (Nasa SP-50, Washington, DC) pp. 425-439 (1964)

[12] H.P. Furth, J. Killeen \& M.N. Rosenbluth, Finite-resistivity instabilities of a sheet pinch, Phys. Fluids 16 459-484 (1963)

[13] E.R. Priest \& T.G. Forbes, New models for fast steady state reconnection, J. Geophys. Res. 91 5579-5588 (1986)

[14] E. Tandberg-Hanssen \& A.G. Emslie, The Physics of Solar Flares, (Cambridge Univ. Press, Cambridge) (1988)

[15] D. Biskamp, Nonlinear Magnetohydrodynamics in Cambridge Monographs on Plasma Physics, Cambridge University Press, Cambridge pp. 127-174 (1993)

[16] E.G. Zweibel \& M. Yamada, Magnetic reconnection in astrophysical and laboratory plasmas, Annu. Rev. Astron. Astrophys. 47 291-332 (2009)

[17] T.J.M. Boyd \& J.J. Sanderson, The Physics of Plasmas(Cambridge University Press, Cambdridge) p. 142-143 (2003)

[18] P.L. Pritchett \& C.C. Wu, The coalescence of magnetic fields, Phys.Fluids 222140 (1979)

[19] M. Aschwanden, The physics of the Solar Corona, An Introduction with Problems and Solutions (Springer-Verlag-Berlin Heidelberg New York) pp. 407-463 (2006)

[20] M. Hesse \& M. Kuznetsova, Particle-in-cell simulations of three-dimensional collisionless magnetic reconnection, Journal of Geophysical Research 106, no A12 29,831-29,841 (2001)

[21] F. Wilson, T. Neukirch, M. Hesse, M.G. Harrison \& C.R. Stark, Particle-in-cell simulations of collisionless magnetic reconnection with a non-uniform guide field, Physics of plasmas $\mathbf{2 3}$ 032302 (2016) 
[22] E.N. Parker, The acceleration of particles to high energy, in proceedings of The Physics of Non-Thermal Radio Sources, G.Setti (ed.), Dordrecht-Reidel Publishing Company, 137 (1976)

[23] H. Dreicer, Electron and ion runaway in a fully ionized gas I, Phys. Rev. 115238 (1959)

[24] H. Dreicer, Electron and ion runaway in a fully ionized gas II, Phys. Rev 117329 (1960)

[25] B. Cerutti, G.R.Werner, D.A. Uzdensky \& M.C Begelman, Simulations of particle acceleration beyond the classical synchrotron burn-off in magnetic reconnection: An explanation for the Crab flares, Astrophys. J. 770147 (2013)

[26] A. Lazarian \& E.T. Vishniac, Reconnection in a weakly stochastic field, ApJ 517700 (1999)

[27] J.W. Armstrong, B.J. Rickett \& S.R. Spangler, Electron density power spectrum in the local interstellar medium, ApJ 443 209-221 (1995)

[28] A Cherpurnov \& A lazarian, Extending the Big Power Law in the Sky with Turbulence Spectra from Wisconson $H_{\alpha}$ Mapper Data, ApJ 710 853-858 (2010)

[29] E. M. de Gouveia dal Pino \& A. Lazarian, Production of the large scale superluminal ejections of the microquasar GRS 1915+105 by violent magnetic reconnection, A\&A 441 p.845853 (2005)

[30] E.M. de Gouveia dal Pino,P.P. Piovezan \& L.H.S. Kadowaki, The role of reconnection on jet/accretion disk systems, A\&A 518 A5 (2010)

[31] G. Kowal, E.M de Gouveia dal Pino \& A. lazarian, magnetohydrodynamic simulations of reconnection and particle acceleration: Three dimensional effects, ApJ 735102 (2011)

[32] G. Kowal, E.M de Gouveia dal Pino \& A. lazarian, Acceleration in turbulence and weakly stochastic reconnection, Phys. Rev. Lett. 108241102 (2012)

[33] M.S Longair, High Energy Astrophysics, Vol 2: Stars and the Interstellar Medium, 2nd ed. Cambridge Univ. Press, Cambridge p. 351 (1994)

[34] D. Giannios, D.A. Uzdensky M.C. Begelman, Fast TeV variability in blazars: jets in a jet, MNRAS 395 L29 (2009)

[35] N.U. Shakura \& R.A. Sunyaev, Black holes in binary systems. Observational appearance, $A \& A 24$ (1997) 337

[36] J.F. Hawley, C.F. Gammie \& S.A. Balbus, Local three dimensional magnetohydrodynamical simulations of accretion discs, ApJ 440742 (1995)

[37] S.A. Balbus \& J.F. Hawley, Instability, Turbulence and enhance transport in accretion disks, Rev. Mod. Phys. 70 pp. 1-52 (1998)

[38] E.P. Velikhov, Stability of an ideally conducting liquid rotating between cylinders in a magnetic field, Sov. Phys. JETP 361389 (1959) 
[39] S. Chandrasekhar, The stability on non-dissipative Couette flow in hydromagnetics, Proc. Nat. Acad. Sci 46 253-257 (1960)

[40] S. Chandrasekhar, Hydrodynamic and hydromagnetic stability, (Dover Publications Inc, New York) pp. 382-427 (1961)

[41] D.L. Coppejans, E.G. Körding, J.C.A. Miller-Jones, M.P. Rupen, C. Knigge, G.R. Sivakovv \& P.J. Groot, Novalike cataclysmic variables are significant radio emitters, MNRAS 451 38013813 (2015)

[42] P.J. Meintjes \& E. Breedt, Magnetic viscocity: Outbursts and outflows in accretion driven systems, in Proceedings of High Energy Astrophysics in Southern Africa: A multi-frequency perspective of new frontiers in high energy astrophysics in Southern Africa (eds. B van Soelen \& P. Meintjes) Mem. S.A. It Vol 86 (1) 89 (2015)

[43] P.J. Meintjes, E. Breedt \& M. Erwee, Magnetized Accretion Discs and the Dwarf Nova Phenomenon, in Proceedings of Frontier Research in Astrophysics (eds. F. Giovanelli, J. Beall, S. Colafeancesco, D. Bisikalo and L. Sabau-Graziati) Proceedings of Science PoS(FRAPWS2014)010 (2015)

[44] P.J. Meintjes, Transient sources in astrphysics: From radio to Gamma-Rays, in proceedings of 3rd Annual Conference on High Energy Astrophysics in Southern Africa (ed. S. Razzaque), Proceedings of Science (2015) PoS(HEASA2015)004 (2015)

[45] E.N. Parker, The dynamical state of the interstellar gas and field, ApJ, 145811 (1966)

[46] M.R. Hayashi, K. Shibata \& R. Matsumoto, X-ray flares and mass outflows driven by magnetic interactionbetween a protostar and its surrounding disc, ApJ 468 L37 (1996)

[47] H. van der Laan A model for variable extragalactic radio sources, Nature 2111131 (1966)

[48] T.S. Bastian, G.A. Dulk \& G. Chanmugam, Radio Flares from AE Aquarii: A Low power Analogue to Cyg X-3, ApJ 324431 (1988)

[49] P.J. Meintjes \& L.A. Venter, Modelling the continuous radio outbursts in AE Aquarii, MNRAS 341891 (2003) 